\title{
Communication Among Agents: A Way To Revise Beliefs In KD45 Kripke Structures ${ }^{1}$
}

\author{
Jean-Marc Tallon* - Jean-Christophe Vergnaud ${ }^{* *}$-Shmuel Zamir*** \\ * EUREQua, CNRS, Université Paris I Panthéon-Sorbonne \\ jmtallon@univ-paris1.fr \\ ** EUREQua, CNRS, Université Paris I Panthéon-Sorbonne \\ vergnaud@univ-paris1.fr \\ *** EUREQua, CNRS, Université Paris I Panthéon-Sorbonne, CREST-LEI, and Cen- \\ ter for the Study of Rationality, Hebrew University \\ zamir@math.huji.ac.il
}

ABSTRACT. We address the issue of belief revision in a multi-agent setting. We represent agents' beliefs in a semantic manner, through a Kripke structure, and model a communication process by which agents communicate their beliefs to one another. We define a revision rule that can be applied even when agents have contradictory beliefs. We study its properties and show that agents need not agree after communicating their beliefs. We finally address the dynamics of revision and show that the order of communication may affect the resulting belief structure.

KEYWORDS: belief revision, KD45, Kripke structure.

\section{Introduction}

Situations in which agents have mistaken beliefs abound. In this paper, we propose a revision rule that specifies how agents' beliefs evolve after communication among themselves has taken place. Specifically, we work with KD45 Kripke structures (e.g., [CHE 80]) and allow agents to communicate (non strategically) their beliefs. The issue is to come up with a rule specifying how initial beliefs that are contradicted by the announcement of some other agent are changed to cope with this contradiction. In the absence of any mistake (i.e., in S5), the process is simple: each agent simply

1. We would like to thank J.Lang, B.Walliser, and an anonymous referee for useful comments. We also thank the audience at RUD2002 and participants at the workshop on Belief Revision, LIP6, U. Paris VI. Financial support from the French Ministry of Research (ACI Cognitique) is gratefully acknowledged.

Journal of Applied Non-Classical Logics. Volume $-n^{\circ} /$ 
drops from his beliefs the ones that are not compatible with the announcements. This yields a new S5 Kripke structure. However, in the presence of mistaken initial beliefs, the rule has to propose a way to correct these beliefs. We proceed in two steps. First, we specify a selection procedure that specifies which initial beliefs the agent retains upon hearing the other's announcement. We do so by defining agent selection functions on the possible worlds. These selection functions allow for the possibility that agents eliminate certain of their initial beliefs. We do not try to ground these selection functions on some rational basis and leave them essentially unconstrained. The only restriction we consider is a minimal consistency requirement which says the following: any state, initially believed possible, that is not contradicted by the announcements is still considered possible after the revision process. We also modify the accessibility relation so that the beliefs of the agents who have announced are now commonly known. We show that this rule is always well defined, in the sense that it leads to a KD45 Kripke structure. We provide conditions under which no revision occurs. We also give conditions on the initial structure that guarantee the emergence of consensus. We next extend this rule to a dynamic setting, in which agents announce and revise their beliefs sequentially. We show that, interestingly, the rule proposed is commutative whenever agents' beliefs are correct (that is, in S5), but that in general, in KD45, the order according to which the agents announce their beliefs might matter. For instance, the final epistemic situation reached is not the same whether all agents announced simultaneously or one at a time. ${ }^{1}$

We consider only a semantic framework. In certain fields such as economics and game theory, the semantic approach is favored while logicians rather prefers to consider the syntax. We will discuss informally along the text the issue of belief revision from a syntaxical point of view. In particular, we will discuss the difficulties about stating some axioms of belief revision in a multi agents situation with respect to the AGM axioms for a single agent's setting. Indeed, while it would be nice to adapt the AGM [ALC 85] axiomatic method to multi-agent belief revision, this is far from being a simple task. ${ }^{2}$ Indeed, belief revision in a multi-agent framework poses not only the problem of integrating new information but also the issue of how agents perceive how other agents will integrate new information. This could lead for instance to violations of AGM's axiom of success.

\section{Minimal Kripke structures: definition and preliminaries}

Let $I=\{1, \ldots, i, \ldots, n\}$ be a finite set of agents and $S$ a set of states of nature (for instance the game that is being played among the agents). A Kripke structure is a representation of agents' beliefs about the state of nature $s$ and about the beliefs of the other agents.

1. The possibility of non commutativity of belief revision has already been noticed in the literature. See [GER 97].

2. For an attempt in that direction, see [BOA 03]. 
DEFINITION 1. - A Minimal Kripke Structure (MKS) is a collection $\left(\Omega, \omega_{0}, s,\left(t_{i}\right)_{i \in I}\right)$, where $\Omega$ is a set, and the following conditions are satisfied:

(i) $s$ is a mapping from $\Omega$ to $S$,

(ii) $\forall i \in I, t_{i}$ is a mapping from $\Omega$ to $2^{\Omega}$,

(iii) $\forall i \in I, \forall \omega \in \Omega, \omega^{\prime} \in t_{i}(\omega) \Rightarrow t_{i}\left(\omega^{\prime}\right)=t_{i}(\omega)$,

(iv) $\omega_{0} \in \Omega$,

(v) There does not exist $\Omega^{\prime} \varsubsetneqq \Omega$ such that $\left(\Omega^{\prime}, \omega_{0},\left.s\right|_{\Omega^{\prime}},\left(\left.t_{i}\right|_{\Omega^{\prime}}\right)_{i \in I}\right)$ satisfies conditions (i) to (iv). ${ }^{3}$

We will refer to an element $\left(\omega ; s(\omega) ; t_{1}(\omega), \ldots, t_{n}(\omega)\right)$ as a state of the world. $\omega$ is the name of the state, $s(\omega)$ is the state of nature in the world $\omega, t_{i}(\omega)$ is the set of states of the world that $i$ considers possible in state $\omega$. Finally, $\omega_{0}$ is the true state of the world. Abusing notation slightly we will denote a state of the world $\omega=$ $\left(s(\omega), t_{1}(\omega), \ldots, t_{n}(\omega)\right)$.

Since we do not require that agents consider $\omega_{0}$ possible, the structures we look at may contain mistaken beliefs. Hence, we place ourselves in the system KD45 rather than S5. Embedded in the definition are several assumptions about the nature of the situations we model. First, we assume a form of consistency of the beliefs: (iii) of the definition implies that beliefs are partitional (i.e., $\left\{t_{i}(\omega)\right\}_{\omega \in \Omega}$ is a partition of $\Omega_{i}=: \cup_{\omega \in \Omega} t_{i}(\omega)$ ). Note however that $\Omega_{i}$ is not necessarily equal to $\Omega$. Second, the true state $\omega_{0}$ is given, since by construction an MKS is a representation of given beliefs (the ones encapsulated in $\omega_{0}$, the other states being part of the description of these beliefs). Third, we assume that the Kripke structure is minimal in the sense that it does not contain a smaller Kripke structure (condition $(v)$ ). This last condition is equivalent to assuming that the system does not contain states that are not deemed possible via a finite sequence of steps of the form "I think that you think that she thinks..." (condition $\left(v^{\prime}\right)$ in Proposition 2 below, which will be used repeatedly in the proofs of this paper.) This does not imply that $\Omega$ is finite.

Proposition 2. $-{ }^{4}$ Let $\left(\Omega, \omega_{0}, s,\left(t_{i}\right)_{i \in I}\right)$ be a collection which satisfies conditions (i) to (iv) of Definition 1. Then condition $(v)$ is equivalent to

$\left(v^{\prime}\right) \forall \omega \in \Omega \backslash\left\{\omega_{0}\right\}$, there exists a finite sequence, $\left\{i_{k}\right\}_{k=1}^{k=r}$ with $i_{k} \in I$ for all $k$ such that $\omega \in t_{i_{1}}\left(t_{i_{2}}\left(\ldots\left(t_{i_{r}}\left(\omega_{0}\right)\right)\right)\right)$ where for any $A \subseteq \Omega, t_{i}(A)=\cup_{\omega \in A} t_{i}(\omega)$.

Condition (v') defines what is often called the generated sub-model. The classical example of the muddy children can be expressed in this formalism.

EXAMPLE 3. - Three children come home after playing in the field. They might have a clean face $(C)$ or a dirty face $(D)$. Each child sees the other two's faces but does not see whether there is mud on her own face. Assume that the three faces are actually

3. $\left.t_{i}\right|_{\Omega^{\prime}}$ is the restriction of $t_{i}$ to $\Omega^{\prime}$, i.e., $\left.t_{i}\right|_{\Omega^{\prime}}: \Omega^{\prime} \rightarrow 2^{\Omega}$ and $\left.t_{i}\right|_{\Omega^{\prime}}(\omega)=t_{i}(\omega)$ for all $\omega \in \Omega^{\prime}$. 4. All proofs are gathered in Appendix B. 
dirty. Denoting the states of nature by $F_{1} F_{2} F_{3}$ where $F_{i} \in\{C, D\}$ is the state of $i$ 's face, we represent this situation by an MKS given by $\Omega=\left\{\omega_{0}, \ldots, \omega_{7}\right\}$ where,

$$
\begin{aligned}
& \omega_{0}=\left(D D D,\left\{\omega_{0}, \omega_{4}\right\},\left\{\omega_{0}, \omega_{2}\right\},\left\{\omega_{0}, \omega_{1}\right\}\right) \\
& \omega_{1}=\left(D D C,\left\{\omega_{1}, \omega_{5}\right\},\left\{\omega_{1}, \omega_{3}\right\},\left\{\omega_{0}, \omega_{1}\right\}\right) \\
& \omega_{2}=\left(D C D,\left\{\omega_{2}, \omega_{6}\right\},\left\{\omega_{0}, \omega_{2}\right\},\left\{\omega_{2}, \omega_{3}\right\}\right) \\
& \omega_{3}=\left(D C C,\left\{\omega_{3}, \omega_{7}\right\},\left\{\omega_{1}, \omega_{3}\right\},\left\{\omega_{2}, \omega_{3}\right\}\right) \\
& \omega_{4}=\left(C D D,\left\{\omega_{0}, \omega_{4}\right\},\left\{\omega_{4}, \omega_{6}\right\},\left\{\omega_{4}, \omega_{5}\right\}\right) \\
& \omega_{5}=\left(C D C,\left\{\omega_{1}, \omega_{5}\right\},\left\{\omega_{5}, \omega_{7}\right\},\left\{\omega_{4}, \omega_{5}\right\}\right) \\
& \omega_{6}=\left(C C D,\left\{\omega_{2}, \omega_{6}\right\},\left\{\omega_{4}, \omega_{6}\right\},\left\{\omega_{6}, \omega_{7}\right\}\right) \\
& \omega_{7}=\left(C C C,\left\{\omega_{3}, \omega_{7}\right\},\left\{\omega_{5}, \omega_{7}\right\},\left\{\omega_{6}, \omega_{7}\right\}\right)
\end{aligned}
$$

The next example illustrates an instance of mistaken beliefs.

EXAMPLE 4. - Let $S=\{\alpha, \beta\}, I=\{1,2\}$ and $\Omega=\left\{\omega_{0}, \omega_{1}, \omega_{2}, \omega_{3}\right\}$ such that:

$$
\begin{aligned}
& \omega_{0}=\left(\alpha,\left\{\omega_{1}, \omega_{2}\right\},\left\{\omega_{3}\right\}\right) \\
& \omega_{1}=\left(\alpha,\left\{\omega_{1}, \omega_{2}\right\},\left\{\omega_{1}, \omega_{2}\right\}\right) \\
& \omega_{2}=\left(\beta,\left\{\omega_{1}, \omega_{2}\right\},\left\{\omega_{1}, \omega_{2}\right\}\right) \\
& \omega_{3}=\left(\beta,\left\{\omega_{3}\right\},\left\{\omega_{3}\right\}\right)
\end{aligned}
$$

To describe the situation which is represented in this structure, let us introduce some elements of syntax. First, for notational simplicity we will denote also by $\alpha, \beta \ldots$ the primitive propositions i.e: considered as a proposition, $\alpha$ means "the nature is in state $\alpha^{\prime \prime}$. We note $\wedge, \vee, \neg$, and $\rightarrow$ for respectively, the and, or, negation and material implication operators. We consider individual belief operators $b_{i}$ and a common belief operator $c b .{ }^{5}$ Therefore, the previous example catches a situation where the proposition $\alpha \wedge b_{1} c b(\alpha \vee \beta) \wedge b_{2} c b \beta$ holds true, that is, in words, a situation where the state of nature is $\alpha$, where agent 1 believes that it is common belief that $\alpha$ or $\beta$ and agent 2 believes that it is common belief that $\beta$.

A given epistemic situation could be captured by MKS that are formally different. This fact is not bothersome in S5, i.e., if agents do not make any mistake. However, as we want to study revision in beliefs when agents potentially have initial mistaken beliefs, we have to make sure that "irrelevant" mistakes can be dropped at the outset so as to focus on beliefs that are mistaken in a meaningful way. A simple intuition of why some mistakes are not meaningful is the following: imagine that $\omega_{0} \notin t_{i}\left(\omega_{0}\right)$.

5. The common belief operator $c b$ has the intuitive meaning that everybody believes that everybody believes...an infinite number of time. Since we do not allow for infinite conjunction, the definition of the common belief operator cannot be defined from the individual belief operators and its properties have to be defined per se. For instance, we have $c b \varphi \rightarrow b_{i} \varphi$ and $c b \varphi \rightarrow b_{i} c b \varphi$ for all $i$ and proposition $\varphi$. See [BON 96]. 
This can reflect two very different situations: either the agent is correct in the sense that in $\omega_{0}$ he believes possible a state $\omega^{\prime}$ which represents the same beliefs as $\omega_{0}$; or the agent is making a mistake in the sense that he is not considering as possible the true state of the world $\omega_{0}$ or any state of the world that represents the same epistemic state.

EXAMPLE 5. - Let $S=\{\alpha\}, I=\{1,2\}$ and consider two MKS:

$\Omega=\left\{\omega_{0}, \omega_{1}\right\}$ such that:

$$
\begin{aligned}
\omega_{0} & =\left(\alpha,\left\{\omega_{1}\right\},\left\{\omega_{1}\right\}\right) \\
\omega_{1} & =\left(\alpha,\left\{\omega_{1}\right\},\left\{\omega_{1}\right\}\right) \\
\text { and } \Omega^{\prime} & =\left\{\omega_{0}^{\prime}\right\} \text { such that: } \\
\omega_{0}^{\prime} & =\left(\alpha,\left\{\omega_{0}^{\prime}\right\},\left\{\omega_{0}^{\prime}\right\}\right)
\end{aligned}
$$

These two MKS represent the same situation: $\alpha$ is the state of nature and it is common belief that $\alpha$ is the state of nature.

A way of getting around this difficulty is to define notions of representation and equivalence of MKS as well as a notion of irreducibility for MKS.

DEFInition 6. - An MKS, $\left(\Omega^{\prime}, \omega_{0}^{\prime}, s^{\prime},\left(t_{i}^{\prime}\right)_{i \in I}\right)$, is a representation of the MKS,

$\left(\Omega, \omega_{0}, s,\left(t_{i}\right)_{i \in I}\right)$, if there exists a mapping $\sigma$ from $\Omega$ to $\Omega^{\prime}$ such that

(i) $\sigma(\Omega)=\Omega^{\prime}$

(ii) $\sigma\left(\omega_{0}\right)=\omega_{0}^{\prime}$

(iii) $s^{\prime} \circ \sigma=s$

(iv) $\forall i \in I, t_{i}^{\prime} \circ \sigma=\sigma \circ t_{i}$.

Definition 7. - Two MKS, $\left(\Omega, \omega_{0}, s,\left(t_{i}\right)_{i \in I}\right)$ and $\left(\Omega^{\prime}, \omega_{0}^{\prime}, s^{\prime},\left(t_{i}^{\prime}\right)_{i \in I}\right)$, are equivalent if they have a common representation, $\left(\Omega^{\prime \prime}, \omega_{0}^{\prime \prime}, s^{\prime \prime},\left(t_{i}^{\prime \prime}\right)_{i \in I}\right)$.

This notion of equivalence corresponds to bisimulation. We now define a notion of redundancy within an MKS.

DEFINITION 8. - Let $\left(\Omega, \omega_{0}, s,\left(t_{i}\right)_{i \in I}\right)$ be an MKS. Two states $\omega_{1}, \omega_{2} \in \Omega$ are said to be identical if there exists an MKS, $\left(\Omega^{\prime}, \omega_{0}^{\prime}, s^{\prime},\left(t_{i}^{\prime}\right)_{i \in I}\right)$ and a mapping $\sigma: \Omega \rightarrow \Omega^{\prime}$ as in Definition 6 such that $\sigma\left(\omega_{1}\right)=\sigma\left(\omega_{2}\right)$.

Two states of the world are thus identical if there exists a representation of the MKS in which these two states are represented by the same state of the world. Our next step is to define irreducible MKS, in which such a problem does not arise.

DEFINITION 9. -

- An MKS, $\left(\Omega, \omega_{0}, s,\left(t_{i}\right)_{i \in I}\right)$ is irreducible if no two distinct states of the world $\omega, \omega^{\prime} \in \Omega$, are identical. 
- An MKS, $\left(\Omega^{\prime}, \omega_{0}^{\prime}, s^{\prime},\left(t_{i}^{\prime}\right)_{i \in I}\right) \quad$ is an irreducible representation of $\left(\Omega, \omega_{0}, s,\left(t_{i}\right)_{i \in I}\right)$ if it is a representation of $\left(\Omega, \omega_{0}, s,\left(t_{i}\right)_{i \in I}\right)$ and it is irreducible.

In the paper we deal exclusively with irreducible MKS. This is without loss of generality as the next proposition makes it clear, since non irreducible MKS always have an irreducible representation.

Proposition 10. - Let $\left(\Omega, \omega_{0}, s,\left(t_{i}\right)_{i \in I}\right)$ be an MKS. Then it has an irreducible representation $\left(\Omega^{\prime}, \omega_{0}^{\prime}, s^{\prime},\left(t_{i}^{\prime}\right)_{i \in I}\right)$ and all its irreducible representations are equivalent.

Finally, we define a notion of correctness for MKS.

Definition 11. - Let $\left(\Omega, \omega_{0}, s,\left(t_{i}\right)_{i \in I}\right)$ be an irreducible MKS. An agent $i \in I$ has correct beliefs if $\omega_{0} \in t_{i}\left(\omega_{0}\right)$. The MKS is correct if all agents have correct beliefs. The MKS is totally correct if $\omega \in t_{i}(\omega)$ for all $\omega \in \Omega$ and all $i \in I .^{6}$

Total correctness amounts to assume S5. Obviously, an MKS can be correct but not totally correct, as illustrated in the following example.

EXAMPLE 12. - Let $S=\{\alpha, \beta\}$ and $I=\{1,2\}$. Consider $\Omega=\left\{\omega_{0}, \omega_{1}\right\}$ where

$$
\begin{aligned}
& \omega_{0}=\left(\alpha,\left\{\omega_{0}\right\},\left\{\omega_{0}, \omega_{1}\right\}\right) \\
& \omega_{1}=\left(\beta,\left\{\omega_{0}\right\},\left\{\omega_{0}, \omega_{1}\right\}\right)
\end{aligned}
$$

\section{Common belief in minimal Kripke structures}

When agents hold mistaken beliefs, they do not necessarily all have the same view of what the model actually is. We introduce here the notion of belief horizon of an agent which is the model the agent has in mind.

DEFINITION 13. - Let $\left(\Omega, \omega_{0}, s,\left(t_{i}\right)_{i \in I}\right)$ be an MKS. The belief horizon of agent $i \in I$, denoted by $B H_{i}\left(\omega_{0}, t\right)$, is the minimal subset $Y$ of $\Omega$ satisfying:

(i) $t_{i}\left(\omega_{0}\right) \subseteq Y$,

(ii) $\forall \omega \in Y, \forall j \in I, t_{j}(\omega) \subseteq Y$.

Thus, $B H_{i}\left(\omega_{0}, t\right)$ is the smallest set such that $i$ believes it and believes that all other agents believe it, believes that others believe that others believe it and so forth. In Example 4, one has $B H_{1}\left(\omega_{0}, t\right)=\left\{\omega_{1}, \omega_{2}\right\}$ and $B H_{2}\left(\omega_{0}, t\right)=\left\{\omega_{3}\right\}$.

Proposition 14. - Let $\left(\Omega, \omega_{0}, s,\left(t_{i}\right)_{i \in I}\right)$ be an MKS. Then,

$$
\Omega=\left\{\omega_{0}\right\} \cup\left(\bigcup_{i \in I} B H_{i}\left(\omega_{0}, t\right)\right)
$$

6. If the MKS considered were not irreducible, the definition should be slightly more general: an MKS is correct if $\forall i \in I$, there exists $\omega \in t_{i}\left(\omega_{0}\right)$, such that $\omega$ and $\omega_{0}$ are identical. When the MKS is irreducible, this definition and Definition 11 coincide. 
Define now the notion of common belief.

Definition 15. - Let $\left(\Omega, \omega_{0}, s,\left(t_{i}\right)_{i \in I}\right)$ be an MKS. An event $E \subseteq \Omega$ is common belief $(C B)$ if for any $r \in \mathbb{N}$ and sequence $\left\{i_{k}\right\}_{k=1}^{k=r}, i_{k} \in I, t_{i_{1}}\left(t_{i_{2}}\left(\ldots\left(t_{i_{r}}\left(\omega_{0}\right)\right)\right) \subseteq\right.$ E.

Note that as an MKS describes a mutual belief structure at a specific, "true", state of the world, common belief is also defined at that state $\omega_{0}$. The following proposition characterizes those events that are common beliefs.

Proposition 16. - Let $\left(\Omega, \omega_{0}, s,\left(t_{i}\right)_{i \in I}\right)$ be an MKS. An event $E \subseteq \Omega$ is common belief if and only if $B H_{i}\left(\omega_{0}, t\right) \subseteq E$ for all $i \in I$.

This notion of common belief is meaningful for the analyst since, according to $i$ 's beliefs, any event containing $B H_{i}\left(\omega_{0}, t\right)$ is $\mathrm{CB}$. As we shall see later, only at the absence of mistakes, $\mathrm{CB}$ events have stronger meaning.

Corollary 17. - Let $\left(\Omega, \omega_{0}, s,\left(t_{i}\right)_{i \in I}\right)$ be an MKS. An event $E \subseteq \Omega$ is common belief if and only if

$$
\cup_{i \in I} B H_{i}\left(\omega_{0}, t\right) \subseteq E \subseteq \Omega=\left\{\omega_{0}\right\} \cup\left(\bigcup_{i \in I} B H_{i}\left(\omega_{0}, t\right)\right)
$$

This corollary establishes that in an MKS, at most two events can be common belief. $\Omega$ is always commonly believed (by construction), while $\Omega \backslash\left\{\omega_{0}\right\}$ is common belief only if the true state $\omega_{0}$ does not belong to the belief horizon of any agent. In other words, $\Omega$ is the only common belief event at $\omega_{0}$ if and only if $\omega_{0}$ is in the belief of at least one agent, that is, if and only if there exists $i$ such that $\omega_{0} \in t_{i}\left(\omega_{0}\right)$.

In syntaxical terms, that means that $c b \varphi$ is true (in the real state $\omega_{0}$ ) if and only if $\varphi$ is true in all the worlds of $B H_{i}\left(\omega_{0}, t\right)$ for all $i$.

\section{Communication and revision in minimal Kripke structures}

We are interested in studying the evolution of beliefs when agents can communicate their beliefs to each other and update accordingly. In this section we provide a rule according to which agents revise their beliefs in a communication process. At this stage of our work, we do not allow agents to announce false (or partly false) or even imprecise beliefs. Thus, the analysis will concentrate on the case in which agents announce truthfully and precisely their beliefs.

DEFINITION 18. - Let $\left(\Omega, \omega_{0}, s,\left(t_{i}\right)_{i \in I}\right)$ be an MKS. A communication is simply a subset $I^{c}$ of $I$, of agents that announce their beliefs (i.e., $\left.\left(t_{i}\left(\omega_{0}\right)\right)_{i \in I^{c}}\right)$.

A communication can be identified by $I^{c} \subseteq I$, the group of agents who announce their true beliefs. We'll refer to it as full communication when $I^{c}=I$. The restriction 
that agents announce precisely their true beliefs can be understood as an assumption that the information revealed can be somehow certified. We will assume in the sequel that it is "common belief" that agents announce precisely their true beliefs. For instance, in Example 4, full communication by agent 1 and 2 means concretely that agent 1 announces publicly that he believes that the state of nature is $\alpha$ or $\beta$, that he believes that this is common belief, while agent 2 announces that he believes it is common belief that the state of nature is $\beta$.

\subsection{Selection functions}

Before introducing the revision rule we propose, we need to add a sort of personal attitude of the agents as part of the data of the model. Assume that given an MKS, $\left(\Omega, \omega_{0}, s,\left(t_{i}\right)_{i \in I}\right)$ and a communication $I^{c}$ we consider the selection functions satisfying the following definition:

DEFINITION 19. - A selection function $f_{i}$ is a mapping from $\Omega$ to $2^{\Omega}$ that satisfies: ${ }^{7}$

(i) $\forall \omega \in \Omega, f_{i}(\omega) \subseteq t_{i}(\omega)$,

(ii) $\forall \omega, \omega^{\prime} \in \Omega$ such that $t_{i}(\omega)=t_{i}\left(\omega^{\prime}\right)$ we have $f_{i}(\omega)=f_{i}\left(\omega^{\prime}\right)$

(iii) Consistency : $\forall \omega \in \Omega$ if $t_{i}(\omega) \cap\left\{\omega^{\prime} \in \Omega \mid t_{j}\left(\omega^{\prime}\right)=t_{j}\left(\omega_{0}\right) \forall j \in I^{c}\right\} \neq \varnothing$ then $f_{i}(\omega)=t_{i}(\omega) \cap\left\{\omega^{\prime} \in \Omega \mid t_{j}\left(\omega^{\prime}\right)=t_{j}\left(\omega_{0}\right) \forall j \in I^{c}\right\}$

The consistency condition states that every state of the world initially deemed to be possibly believed by $i$ (i.e., states that are in $\Omega_{i}$ ) and that explains (is compatible with) the others' announcements should be kept and furthermore, if there exist such states, only these states should be kept.

A particular selection function which fits the definition is the following: $\forall \omega \in \Omega$

$$
\begin{aligned}
f_{i}(\omega) & =t_{i}(\omega) \cap\left\{\omega^{\prime} \in \Omega \mid t_{j}\left(\omega^{\prime}\right)=t_{j}\left(\omega_{0}\right) \forall j \in I^{c}\right\} \text { if it is not empty } \\
& =t_{i}(\omega) \text { otherwise }
\end{aligned}
$$

This selection function corresponds to a conservative attitude: if the initial beliefs of an agent were proven false by the announcement, then the agent keeps all the possible world he initially believed in.

The revision rule we are about to introduce is based on these selection functions and on the assumption that, loosely speaking, they are commonly believed by all agents so as to enable interactive reasoning about mutual beliefs. It should be noted that the function $f_{i}$ is defined on $\Omega$, which does not, in general, coincide with $i$ 's belief horizon. This is important since $j$ might (mistakenly) believe that $i$ might believe possible states that are not in $i$ 's belief horizon. Hence, $j$ needs to know how to revise

7. Rigorously, one should index a selection function by the communication, that is, $f_{i}^{I^{c}}$. For sake of simplicity we drop the reference to the communication and simply write $f_{i}$. 
$i$ 's beliefs in these worlds and the selection function precisely tells him how to do so. Implicit in the fact that the selection function for agent $i$ is defined on all of $\Omega$ is the idea that all agents agree on how to revise $i$ 's beliefs. If states $\omega$ and $\omega^{\prime}$ belong to both $j$ and $j^{\prime}$ 's belief horizon, then these two agents agree on which states are kept according to $i$ 's selection function. Furthermore, this fact is commonly believed by all agents. Hence, we'll make the maintained implicit assumption that given an MKS, $\left(\Omega, \omega_{0}, s,\left(t_{i}\right)_{i \in I}\right)$ and a communication, all selection functions $f_{i}$ are commonly believed by all agents.

\subsection{Revision rule: definition and example}

We now propose a revision rule that copes with announcements contradicting initial beliefs. We first define the rule and then illustrate it via a few examples. Given an MKS and a communication, the revision rule captures two elements: (i) each agent $i$ retains all states of the world according to the selection function $f_{i}$ and this selection process is commonly believed among the agent, (ii) all announcements made become common beliefs.

Definition 20. - Let $\left(\Omega, \omega_{0}, s,\left(t_{i}\right)_{i \in I}\right)$ be an irreducible MKS, $I^{c}$ a communication, and $\left(f_{i}\right)_{i \in I}$ be selection functions. ${ }^{8}$ The revision of $\left(\Omega, \omega_{0}, s,\left(t_{i}\right)_{i \in I}\right)$ is $\left(\Omega^{c}, \omega_{0}, s,\left(t_{i}^{c}\right)_{i \in I}\right)$ where $t_{i}^{c}($.$) is defined as follows:$

$-\forall \omega \in \Omega, \forall i \in I \backslash I^{c}, t_{i}^{c}(\omega)=f_{i}(\omega)$

$-\forall \omega \in \Omega, \forall i \in I^{c}, t_{i}^{c}(\omega)=f_{i}\left(\omega_{0}\right)$

and $\Omega^{c}=\left\{\omega_{0}\right\} \cup\left(\cup_{i \in I} B H_{i}\left(\omega_{0}, t^{c}\right)\right)$

REMARK 21. - There is a slight abuse of notation in the previous definition, as $\Omega^{c}$ is defined via belief horizons that are only defined once $\Omega^{c}$ is given.

To understand the logic of the revision rule we propose, let us examine in details the following example.

EXAMPLE 22. - (Example 4 continued) Consider first a simple communication by agent 2 . For agent 1 there are three possible selection functions corresponding to the three possible non-empty subset of $\left\{\omega_{1}, \omega_{2}\right\}$ while the selection function $f_{2}$ is simply equal to $t_{2}$. Consider for instance the "conservative" selection function for $f_{1}$, that is: $f_{1}\left(\omega_{0}\right)=f_{1}\left(\omega_{1}\right)=\left\{\omega_{1}, \omega_{2}\right\}$ and $f_{1}\left(\omega_{3}\right)=\left\{\omega_{3}\right\}$.

The revision rule leads to the following MKS:

$$
\begin{aligned}
& \omega_{0}=\left(\alpha,\left\{\omega_{1}, \omega_{2}\right\},\left\{\omega_{3}\right\}\right) \\
& \omega_{1}=\left(\alpha,\left\{\omega_{1}, \omega_{2}\right\},\left\{\omega_{3}\right\}\right) \\
& \omega_{2}=\left(\beta,\left\{\omega_{1}, \omega_{2}\right\},\left\{\omega_{3}\right\}\right)
\end{aligned}
$$

8. We define the revision rule only for irreducible MKS, since otherwise the outcome of the revision process might depend on the representation used. 
$\omega_{3}=\left(\beta,\left\{\omega_{3}\right\},\left\{\omega_{3}\right\}\right)$

It is easy to check that in the above MKS, states $\omega_{0}$ and $\omega_{1}$ actually express the same hierarchy of beliefs and hence, it can be reduced to the following MKS:

$$
\begin{aligned}
& \omega_{0}=\left(\alpha,\left\{\omega_{0}, \omega_{2}\right\},\left\{\omega_{3}\right\}\right) \\
& \omega_{2}=\left(\beta,\left\{\omega_{0}, \omega_{2}\right\},\left\{\omega_{3}\right\}\right) \\
& \omega_{3}=\left(\beta,\left\{\omega_{3}\right\},\left\{\omega_{3}\right\}\right)
\end{aligned}
$$

Therefore, in syntaxical terms we start from a situation where the proposition $\alpha \wedge$ $b_{1} c b(\alpha \vee \beta) \wedge b_{2} c b \beta$ is true (the state of nature is $\alpha$, agent 1 believes that it is common belief that $\alpha$ or $\beta$ and agent 2 believes that it is common belief that $\beta$ ) to a situation where $\alpha \wedge b_{1}(\alpha \vee \beta) \wedge b_{1} b_{2} c b \beta \wedge b_{2} c b \beta$ is true, that is the state of nature is $\alpha$, agent 1 believes $\alpha$ or $\beta$, believes that agent 2 believes that it is common belief that $\beta$ and agent 2 still believes that it is common belief that $\beta$.

The result seems intuitive:

- there is no reason why agent 2 should change his beliefs since he received no announcement and he believes that his own announcements brings no news to agent 1 ,

- agent 1 who discovered that he was wrong about agent 2's beliefs, simply takes them into account in order to modify his own beliefs.

In fact, this case is a very special case which is no more than a single agent revision process: the content of the announcement (i.e: the proposition $b_{2} c b \beta$ ) is taken literally in the revised beliefs of agent 1 . This does not have to be the case in general (even in the case where there is only one agent who announces) as we will see below.

Consider now full communication and let us use the "conservative" selection functions, that is: $f_{1}\left(\omega_{0}\right)=f_{1}\left(\omega_{1}\right)=f_{1}\left(\omega_{2}\right)=f_{2}\left(\omega_{1}\right)=f_{2}\left(\omega_{2}\right)=\left\{\omega_{1}, \omega_{2}\right\}$ and $f_{1}\left(\omega_{3}\right)=f_{2}\left(\omega_{0}\right)=f_{2}\left(\omega_{3}\right)=\left\{\omega_{3}\right\}$.

The revision rule leads to the following MKS:

$$
\begin{aligned}
& \omega_{0}=\left(\alpha,\left\{\omega_{1}, \omega_{2}\right\},\left\{\omega_{3}\right\}\right) \\
& \omega_{1}=\left(\alpha,\left\{\omega_{1}, \omega_{2}\right\},\left\{\omega_{3}\right\}\right) \\
& \omega_{2}=\left(\beta,\left\{\omega_{1}, \omega_{2}\right\},\left\{\omega_{3}\right\}\right) \\
& \omega_{3}=\left(\beta,\left\{\omega_{1}, \omega_{2}\right\},\left\{\omega_{3}\right\}\right)
\end{aligned}
$$

which can be reduced to the following MKS:

$$
\begin{aligned}
& \omega_{0}=\left(\alpha,\left\{\omega_{0}, \omega_{2}\right\},\left\{\omega_{2}\right\}\right) \\
& \omega_{2}=\left(\beta,\left\{\omega_{0}, \omega_{2}\right\},\left\{\omega_{2}\right\}\right)
\end{aligned}
$$


The final situation is a situation where $\alpha \wedge c b\left(b_{1}(\alpha \vee \beta) \wedge b_{2} \beta\right)$ is true, that is the state of nature is $\alpha$ and it is commonly believed that agent 1 believes $\alpha$ or $\beta$ and agent 2 believes $\beta$.

Note that contrarily to the previous single announcement, agent 1 does not simply incorporate in his beliefs agent 2's announcement. Indeed, for instance, the fact that agent 2 believes that agent 1 believes $\beta$ (i.e., $b_{2} b_{1} \beta$ ) is no longer true since agent 1 has announced $b_{1}(\alpha \vee \beta)$ and therefore we can not consider that agent 1 will believe that agent 2 believes that agent 1 believes $\beta$ (i.e., $b_{1} b_{2} b_{1} \beta$ ). Hence starting from the announcements, agents are deducing new beliefs by calculating how the others are deducing their new beliefs... We do not try to formalize the detail of this crossdeduction process and the rule we propose can be seen as a short cut for this process. The result it gives is a rather natural one.

This example illustrates some difficulties with transposing AGM's axiom of success in a multi-agent setting. If one takes that beliefs are revised by the announcements of both agents, i.e., $b_{1} c b(\alpha \vee \beta) \wedge b_{2} c b \beta$, then this announcement is not true any longer in the revised system.

Next, let us still consider full communication but let us change agent 1's selection function: $f_{1}\left(\omega_{0}\right)=f_{1}\left(\omega_{1}\right)=f_{1}\left(\omega_{2}\right)=\left\{\omega_{1}\right\}$ and $f_{1}\left(\omega_{3}\right)=\left\{\omega_{3}\right\}$, maintaining $f_{2}$ as before.

Then we get the following MKS:

$$
\begin{aligned}
& \omega_{0}=\left(\alpha,\left\{\omega_{1}\right\},\left\{\omega_{3}\right\}\right) \\
& \omega_{1}=\left(\alpha,\left\{\omega_{1}\right\},\left\{\omega_{3}\right\}\right) \\
& \omega_{2}=\left(\beta,\left\{\omega_{1}\right\},\left\{\omega_{3}\right\}\right) \\
& \omega_{3}=\left(\beta,\left\{\omega_{1}\right\},\left\{\omega_{3}\right\}\right)
\end{aligned}
$$

which can be reduced to the following MKS:

$$
\begin{aligned}
& \omega_{0}=\left(\alpha,\left\{\omega_{0}\right\},\left\{\omega_{2}\right\}\right) \\
& \omega_{2}=\left(\beta,\left\{\omega_{0}\right\},\left\{\omega_{2}\right\}\right)
\end{aligned}
$$

The final situation is a situation where $\alpha \wedge c b\left(b_{1} \alpha \wedge b_{2} \beta\right)$ is true, that is the true state of nature is $\alpha$ and it is commonly believed that agent 1 believes $\alpha$ and agent 2 believes $\beta$. Compared to the "conservative selection function" case, we see that agent 1 has also revised his beliefs on the state of nature, although there is not much intuition as to why agent 1 should deduce that $\beta$ is not the case. Perhaps, it is reasonable to impose more restrictive conditions on the selection function. But it is not clear which kind of restriction to impose. Indeed, in more complex examples, it is not clear why we should only restrict our attention to "conservative selection functions".

Finally, we illustrate on that example what would possibly happen when agents communicate in a less precise manner than the one assumed so far. Consider for instance the case in which agent 1 announces simply that he believes $\alpha$ or $\beta$ (i.e., 
$\left.b_{1}(\alpha \vee \beta)\right)$. The event in which this is true is $\left\{\omega_{0}, \omega_{1}, \omega_{2}\right\}$. It seems natural to think that beliefs will change in the following manner: whenever we had $\alpha \wedge b_{1}[c b(\alpha \vee$ $\beta)] \wedge b_{2} c b \beta$, we now have $\alpha \wedge b_{1}[c b(\alpha \vee \beta)] \wedge b_{2} c b\left[b_{1}(\alpha \vee \beta) \wedge b_{2} \beta\right]$. In our semantic approach, the MKS representing this change would be:

$$
\begin{aligned}
& \omega_{0}=\left(\alpha,\left\{\omega_{1}, \omega_{2}\right\},\left\{\omega_{3}\right\}\right) \\
& \omega_{1}=\left(\alpha,\left\{\omega_{1}, \omega_{2}\right\},\left\{\omega_{1}, \omega_{2}\right\}\right) \\
& \omega_{2}=\left(\beta,\left\{\omega_{1}, \omega_{2}\right\},\left\{\omega_{1}, \omega_{2}\right\}\right) \\
& \omega_{3}=\left(\beta,\left\{\omega_{3}, \omega_{4}\right\},\left\{\omega_{3}\right\}\right) \\
& \omega_{4}=\left(\alpha,\left\{\omega_{3}, \omega_{4}\right\},\left\{\omega_{4}\right\}\right)
\end{aligned}
$$

It is however not clear what the general revision rule should be when faced with this type of partial communication. Previous work (see [GER 97]) addressed this question for general type of communication. They however do not consider Kripke semantics, but a representation based on non-well-founded sets. This means, loosely speaking, that they tolerate empty belief sets when there exists some contradictions: agents do not attempt to solve the inconsistencies they face.

\subsection{Revision rule: agreement and consensus}

Before studying properties of the revision rule, we check that it is well defined.

PROPOSITION 23. -

Let $\left(\Omega, \omega_{0}, s,\left(t_{i}\right)_{i \in I}\right)$ be an irreducible MKS. Then, $\left(\Omega^{c}, \omega_{0}, s,\left(t_{i}^{c}\right)_{i \in I}\right)$ is a MKS.

We now seek to characterize conditions under which the revision leads to different forms of agreements among agents. This requires making a detour via the definition and characterization of common S-beliefs systems, in which agents' beliefs about the state of nature are common belief.

For a given MKS, $\left(\Omega, \omega_{0}, s,\left(t_{i}\right)_{i \in I}\right)$ define the S-belief to be the event

$$
S B\left(\omega_{0}, t\right)=\left\{\omega \in \Omega \mid s\left(t_{i}(\omega)\right)=s\left(t_{i}\left(\omega_{0}\right)\right) \forall i \in I\right\}
$$

The $S$-belief is the event "for all $i \in I$, agent $i$ believes that the state of nature is in $s\left(t_{i}\left(\omega_{0}\right)\right)$ ". In other words, $S B\left(\omega_{0}, t\right)$ is the subset of $\Omega$ in which the first level beliefs about $S$ are as those in $\omega_{0}$, i.e., the beliefs in the true state. We define now a special case of belief systems, where the first level beliefs about $S$ are common beliefs.

DEFINITION 24. - An MKS, $\left(\Omega, \omega_{0}, s,\left(t_{i}\right)_{i \in I}\right)$ is a common S-belief system

(henceforth CSBS) if $S B\left(\omega_{0}, t\right)$ is common belief.

In a CSBS, the agents' beliefs about the state of nature are common beliefs. Agents need not agree in a CSBS. It is thus possible to represent situations in which agents' disagreement is common belief. Example 12 is an instance of such a situation: 1 
believes $\alpha, 2$ believes $\alpha$ or $\beta$ and this is common belief, i.e., agents disagree and this disagreement is common belief. We now establish properties about the degree to which agents agree after communication and revision have occurred. When all agents communicate, the revision leads to a situation in which beliefs about the state of nature are common belief. When agents still disagree about the state of nature, this models situation in which this disagreement is common belief. Such a case is illustrated in Example 22 above.

Proposition 25. - Let $\left(\Omega, \omega_{0}, s,\left(t_{i}\right)_{i \in I}\right)$ be an irreducible MKS. Then, $\left(\Omega^{c}, \omega_{0}, s,\left(t_{i}^{c}\right)_{i \in I}\right)$ is a CSBS whenever $I^{c}=I$.

When the initial MKS is already a CSBS, that is, when the beliefs about the state of nature of all agents are common belief, then communication does not lead to any further revision.

Proposition 26. - Let $\left(\Omega, \omega_{0}, s,\left(t_{i}\right)_{i \in I}\right)$ be an irreducible CSBS. Then, $\left(\Omega^{c}, \omega_{0}, s,\left(t_{i}^{c}\right)_{i \in I}\right)=\left(\Omega, \omega_{0}, s,\left(t_{i}\right)_{i \in I}\right)$

The notion of CSBS does not entail a strong notion of agreement since indeed, disagreement can be common belief. A particular case of a CSBS is when the first level beliefs of all agents are the same, and thus $t_{i}(\omega)=t_{j}(\omega)$ for all $i, j \in I$ and all $\omega \in \Omega$. This represents a situation of consensus, when all agents have the same beliefs.

Definition 27. - An irreducible MKS, $\left(\Omega, \omega_{0}, s,\left(t_{i}\right)_{i \in I}\right)$ is consensual if for all $i, j \in I, t_{i}\left(\omega_{0}\right)=t_{j}\left(\omega_{0}\right) .^{9}$

We now give a sufficient condition that entails that revision leads to a consensual MKS.

Proposition 28. - Let $\left(\Omega, \omega_{0}, s,\left(t_{i}\right)_{i \in I}\right)$ be an irreducible MKS and let $I^{c}=$ I. Assume $\left\{\omega \in \Omega \mid t_{j}(\omega)=t_{j}\left(\omega_{0}\right) \forall j \in I\right\} \cap t_{i}\left(\omega_{0}\right) \neq \emptyset$ for all $i \in I$. Then, $\left(\Omega^{c}, \omega_{0}, s,\left(t_{i}^{c}\right)_{i \in I}\right)$ is consensual if and only if

$$
\left\{\omega \in \Omega \mid t_{j}(\omega)=t_{j}\left(\omega_{0}\right) \forall j \in I\right\} \cap t_{i}\left(\omega_{0}\right)=\cap_{i \in I} t_{i}\left(\omega_{0}\right)
$$

This proposition establishes necessary and sufficient conditions for consensus to emerge in the case in which the announcements entail no contradiction. A direct corollary is that consensus is implied by total correctness.

COROLlary 29. - Let $\left(\Omega, \omega_{0}, s,\left(t_{i}\right)_{i \in I}\right)$ be an irreducible MKS and assume it is totally correct. Assume further that $I^{c}=I$. Then, $\left(\Omega^{c}, \omega_{0}, s,\left(t_{i}^{c}\right)_{i \in I}\right)$ is consensual.

9. Recall that the MKS we are interested in are irreducible. If the MKS is not irreducible then the definition of consensus needs to be modified: an MKS that has a representation that is consensual would then be deemed "consensual" itself. 


\subsection{Revision rule: dynamics}

We now extend the static framework considered so far to study situations in which announcements are made sequentially. A communication sequence of length $T$, is the specification of a sequence of sets $\left\{I_{\tau}^{c}\right\}_{\tau=1, \ldots, T}$. At each stage $\tau$, agents in $I_{\tau}^{c}$ announce precisely their true beliefs. We'll say that the communication is exhaustive if $\cup_{\tau=1, \ldots, T} I_{\tau}^{c}=I$, i.e., if all agents announce at some point in time. One can also easily adapt the definition of the selection function to take into account this temporal aspect (it is enough to have selection functions indexed by $\tau$ ).

In view of Proposition 23, that asserts that the revision of an MKS is a well defined MKS, the sequential rule of revision in that case is a straightforward extension of the revision rule proposed in Definition 20. This rule is implemented at each stage, yielding an MKS at stage $\tau$ denoted $\Omega_{\tau}^{c}$. Recall however that, without further restrictions on agents' selection function , the revision rule has to be applied to MKS that are irreducible. Hence, if at the end of any given stage, the resulting MKS is not irreducible, then we replace it by one of its irreducible representations before proceeding to the next round of announcement/revision. In this process, we always make sure that the labelling of the true state remains $\omega_{0}$ at all stages. The revision process is well defined in the sense that it does not depend on the choice of the irreducible representation (see Proposition 37 in Appendix A).

Of particular interest in this dynamic setting are first whether agreement is eventually reached and second, whether the order of the announcements (who announces when) might matter for the situation eventually reached. We answer these two questions affirmatively.

Proposition 30. - Let $\left(\Omega, \omega_{0}, s,\left(t_{i}\right)_{i \in I}\right)$ be an irreducible MKS and assume the communication $\left(I_{\tau}^{c}\right)_{\tau=1, \ldots, T}$ is exhaustive, then $\left(\Omega_{T}^{c}, \omega_{0}, s,\left(t_{i, T}^{c}\right)_{i \in I}\right)$ is a CSBS.

The revision process ends when the smallest $k$ such that $\cup_{\tau=1, \ldots, k} I_{\tau}^{c}=I$ is reached. Hence, we established that convergence occurs and at the point of convergence, beliefs about the state of nature are common beliefs (but might be different). The following proposition shows that there is no loss of generality in restricting attention to communication in which an agent announces his beliefs only once, i.e., having an agent announcing his beliefs at two different stages of the communication is useless.

Proposition 31. - Let $\left(\Omega, \omega_{0}, s,\left(t_{i}\right)_{i \in I}\right)$ be an irreducible $M K S,\left(I_{\tau}^{c}\right)_{\tau=1, \ldots, T}$ be a communication, and $\left(f_{i, \tau}\right)_{\tau=1, \ldots, T}$ selection functions. Define the communication $\left(\bar{I}_{\tau}^{c}\right)_{\tau=1, \ldots, T}$ by $\bar{I}_{1}^{c}=I_{1}^{c}$ and $\bar{I}_{\tau}^{c}=I_{\tau}^{c} \backslash \cup_{\tau^{\prime}<\tau} I_{\tau^{\prime}}^{c}$ for $\tau=2, \ldots, T$. Then, if $\left(\Omega_{\tau}^{c}, \omega_{0}, s,\left(t_{i, \tau}^{c}\right)_{i \in I}\right)_{\tau=1, \ldots, T}$ is a sequence of revised MKS after communication $I^{c}$, then, it is also a sequence of revised MKS after communication $\bar{I}^{c}$.

The next point we address is whether the order of announcements matters and show that it does not if the MKS is totally correct, but might otherwise. 
Proposition 32. - Let $\left(\Omega, \omega_{0}, s,\left(t_{i}\right)_{i \in I}\right)$ be an irreducible totally correct MKS. Consider two sequential communications $\left(I_{\tau}^{c}\right)_{\tau=1, \ldots, T}$ and $\left(\bar{I}_{\tau}^{c}\right)_{\tau=1, \ldots, \bar{T}}$ of length $T$ and $\bar{T}$ respectively, such that $\cup_{\tau=1, \ldots, T} I_{\tau}^{c}=\cup_{\tau=1, \ldots, \bar{T}} \bar{I}_{\tau}^{c}$. Then, the revision rule leads to two equivalent $M K S$.

The proposition provides a rather strong sufficient condition (that the MKS is totally correct) under which the order of announcement does not matter. This sufficient condition can be relaxed but not much. In Example 33, it is shown that as soon as one has to cope with contradictions, the order matters. One may wonder whether commutativity holds when there is no contradiction. Example 34 is a case of a correct MKS in which the final beliefs depend on whether the agents announce simultaneously or sequentially. In this case, the order does matter. This points out the fact that as soon as we depart from MKS that satisfies S5, that is situation where all agents satisfy the truth axiom and it is common belief that all agents satisfy the truth axiom, then the order does matter. In Example 34, all agents satisfy the truth axiom but it is not common belief that this is the case. Therefore, in sequential announcement, agents believe that other agents may have to deal with contradictions and this makes the difference.

EXAMPLE 33. - Let $S=\{\alpha, \beta, \gamma\}$ and $I=\{1,2,3\}$. Consider $\Omega=\left\{\omega_{0}, \omega_{1}, \omega_{2}\right\}$ where

$$
\begin{aligned}
& \omega_{0}=\left(\alpha,\left\{\omega_{1}, \omega_{2}\right\},\left\{\omega_{0}, \omega_{1}\right\},\left\{\omega_{0}, \omega_{2}\right\}\right) \\
& \omega_{1}=\left(\beta,\left\{\omega_{1}, \omega_{2}\right\},\left\{\omega_{0}, \omega_{1}\right\},\left\{\omega_{1}\right\}\right) \\
& \omega_{2}=\left(\gamma,\left\{\omega_{1}, \omega_{2}\right\},\left\{\omega_{2}\right\},\left\{\omega_{0}, \omega_{2}\right\}\right)
\end{aligned}
$$

- First consider the case where there is only one round of announcement and $I^{c}=$ $\{1,2,3\}$. Given this announcement, there is only one possible selection function for each agent 2 and 3 ,

$$
f_{2}\left(\omega_{0}\right)=f_{2}\left(\omega_{1}\right)=f_{3}\left(\omega_{0}\right)=f_{3}\left(\omega_{2}\right)=\left\{\omega_{0}\right\}, f_{2}\left(\omega_{2}\right)=\left\{\omega_{2}\right\}, f_{3}\left(\omega_{1}\right)=\left\{\omega_{1}\right\}
$$

while there are three possible selection functions for agent 1

(a) $f_{1}\left(\omega_{0}\right)=f_{1}\left(\omega_{1}\right)=f_{1}\left(\omega_{2}\right)=\left\{\omega_{1}, \omega_{2}\right\}$

(b) $f_{1}\left(\omega_{0}\right)=f_{1}\left(\omega_{1}\right)=f_{1}\left(\omega_{2}\right)=\left\{\omega_{1}\right\}$

(c) $f_{1}\left(\omega_{0}\right)=f_{1}\left(\omega_{1}\right)=f_{1}\left(\omega_{2}\right)=\left\{\omega_{2}\right\}$

We give the outcome of the revision in these three cases:

- Case (a): the revision rule yields the following MKS

$\omega_{0}=\left(\alpha,\left\{\omega_{1}, \omega_{2}\right\},\left\{\omega_{0}\right\},\left\{\omega_{0}\right\}\right)$

$\omega_{1}=\left(\beta,\left\{\omega_{1}, \omega_{2}\right\},\left\{\omega_{0}\right\},\left\{\omega_{0}\right\}\right)$

$\omega_{2}=\left(\gamma,\left\{\omega_{1}, \omega_{2}\right\},\left\{\omega_{0}\right\},\left\{\omega_{0}\right\}\right)$

- Case (b): the revision rule yields the following MKS

$\omega_{0}=\left(\alpha,\left\{\omega_{1}\right\},\left\{\omega_{0}\right\},\left\{\omega_{0}\right\}\right)$

$\omega_{1}=\left(\beta,\left\{\omega_{1}\right\},\left\{\omega_{0}\right\},\left\{\omega_{0}\right\}\right)$

- Case (c): the revision rule yields the following MKS 


$$
\begin{aligned}
& \omega_{0}=\left(\alpha,\left\{\omega_{2}\right\},\left\{\omega_{0}\right\},\left\{\omega_{0}\right\}\right) \\
& \omega_{2}=\left(\gamma,\left\{\omega_{2}\right\},\left\{\omega_{0}\right\},\left\{\omega_{0}\right\}\right)
\end{aligned}
$$

- Consider now the case where 1 and 2 announce first, revision occurs, and then 3 announces, that is, $I_{1}^{c}=\{1,2\}$ and $I_{2}^{c}=\{3\}$. In the first round, the only possible selection functions are that $f_{1}\left(\omega_{0}\right)=f_{1}\left(\omega_{1}\right)=f_{1}\left(\omega_{2}\right)=\left\{\omega_{1}\right\}$ for agent 1, $f_{2}\left(\omega_{0}\right)=f_{2}\left(\omega_{1}\right)=\left\{\omega_{0}, \omega_{1}\right\}, f_{2}\left(\omega_{2}\right)=\left\{\omega_{2}\right\}$ for agent 2 and $f_{3}\left(\omega_{0}\right)=f_{3}\left(\omega_{2}\right)=$ $\left\{\omega_{0}\right\}, f_{3}\left(\omega_{1}\right)=\left\{\omega_{1}\right\}$ for agent 3 . Thus, the revised MKS after the first round is given by

$$
\begin{aligned}
& \omega_{0}=\left(\alpha,\left\{\omega_{1}\right\},\left\{\omega_{0}, \omega_{1}\right\},\left\{\omega_{0}\right\}\right) \\
& \omega_{1}=\left(\beta,\left\{\omega_{1}\right\},\left\{\omega_{0}, \omega_{1}\right\},\left\{\omega_{1}\right\}\right)
\end{aligned}
$$

The same type of computation after 3's announcement yields:

$$
\begin{aligned}
& \omega_{0}=\left(\alpha,\left\{\omega_{1}\right\},\left\{\omega_{0}\right\},\left\{\omega_{0}\right\}\right) \\
& \omega_{1}=\left(\beta,\left\{\omega_{1}\right\},\left\{\omega_{0}\right\},\left\{\omega_{0}\right\}\right)
\end{aligned}
$$

- Finally, consider the case where 1 and 3 announce first, revision occurs and then 2 announces, that is, $I_{1}^{c}=\{1,3\}$ and $I_{2}^{c}=\{2\}$. Here again, selection functions are uniquely determined and we obtain after the first round:

$$
\begin{aligned}
& \omega_{0}=\left(\alpha,\left\{\omega_{2}\right\},\left\{\omega_{0}\right\},\left\{\omega_{0}, \omega_{2}\right\}\right) \\
& \omega_{2}=\left(\gamma,\left\{\omega_{2}\right\},\left\{\omega_{2}\right\},\left\{\omega_{0}, \omega_{2}\right\}\right)
\end{aligned}
$$

and finally we have, after 2's announcement:

$$
\begin{aligned}
& \omega_{0}=\left(\alpha,\left\{\omega_{2}\right\},\left\{\omega_{0}\right\},\left\{\omega_{0}\right\}\right) \\
& \omega_{2}=\left(\gamma,\left\{\omega_{2}\right\},\left\{\omega_{0}\right\},\left\{\omega_{0}\right\}\right)
\end{aligned}
$$

Thus, we end up with different MKS according to the order of announcements.

In this example, observe that non-commutativity does not come from possible inconsistencies in the selection functions. Non-commutativity comes from the fact that agents' revisions are done sequentially without keeping track of the reason why they changed their initial beliefs to begin with. This absence of memory explains why, in the sequential process in which 1 and 3 announce first and 2 second, 1 does not reconsider the elimination of $\omega_{1}$ (made upon 3's announcement) when 2 announces in the second stage. In the next example, the outcome of the revision process depends on the sequence of announcements although the MKS is initially correct (but not totally correct).

ExAmple 34. - Let $S=\{\alpha, \beta\}$ and $I=\{1,2\}$. Consider $\Omega=\left\{\omega_{0}, \omega_{1}, \omega_{2}\right\}$ where

$$
\begin{aligned}
& \omega_{0}=\left(\alpha,\left\{\omega_{0}, \omega_{1}\right\},\left\{\omega_{0}\right\}\right) \\
& \omega_{1}=\left(\beta,\left\{\omega_{0}, \omega_{1}\right\},\left\{\omega_{2}\right\}\right) \\
& \omega_{2}=\left(\alpha,\left\{\omega_{2}\right\},\left\{\omega_{2}\right\}\right)
\end{aligned}
$$

- First consider the case where agents announce simultaneously. The only possible selection function for agent 1 is $f_{1}\left(\omega_{0}\right)=f_{1}\left(\omega_{1}\right)=\left\{\omega_{0}\right\}, f_{1}\left(\omega_{2}\right)=\left\{\omega_{2}\right\}$. Then we 
obtain the following MKS:

$$
\omega_{0}=\left(\alpha,\left\{\omega_{0}\right\},\left\{\omega_{0}\right\}\right)
$$

- Consider now the case where $I_{1}^{c}=\{1\}$ and $I_{2}^{c}=\{2\}$. After the first announcement, we obtain the following MKS:

$$
\begin{aligned}
& \omega_{0}=\left(\alpha,\left\{\omega_{0}, \omega_{1}\right\},\left\{\omega_{0}\right\}\right) \\
& \omega_{1}=\left(\beta,\left\{\omega_{0}, \omega_{1}\right\},\left\{\omega_{2}\right\}\right) \\
& \omega_{2}=\left(\alpha,\left\{\omega_{0}, \omega_{1}\right\},\left\{\omega_{2}\right\}\right)
\end{aligned}
$$

Before proceeding to the second round of revision, observe that the MKS after the first round is not irreducible since state 0 and 2 are identical. Hence, it has an irreducible representation:

$$
\begin{aligned}
& \omega_{0}=\left(\alpha,\left\{\omega_{0}, \omega_{1}\right\},\left\{\omega_{0}\right\}\right) \\
& \omega_{1}=\left(\beta,\left\{\omega_{0}, \omega_{1}\right\},\left\{\omega_{0}\right\}\right)
\end{aligned}
$$

Now, consider the second step, in which 2 announces his beliefs, i.e., $\omega_{0}$. This does not lead to any further revision.

Hence, the MKS we end up with is different from the one in which both agents were making their announcements simultaneously, showing that the order of these announcements matters, even though the initial MKS was correct.

Another feature of the example is to show that although the agents reach a consensus in the simultaneous case (the conditions of Proposition 28 are satisfied), the sequential revision does not lead to consensus.

The two previous examples show that the revision process is not necessarily commutative, unless the initial MKS is well behaved (i.e., totally correct) as established in Proposition 32. This points out a few interesting issues. First, the non commutativity is not directly linked to the procedure we adopted to treat announcements that are in contradiction with the initial beliefs of the agents. Indeed, in Example 34, the two sequential processes studied do not entail any contradiction: in both cases, the announcements made in the first round are compatible with part of the initial beliefs. Thus, agents only keep those states that are exactly compatible with the announcements. Second, non-commutativity of the revision procedure arises because agents treat each new MKS afresh, without keeping track of how they arrived at it. In that respect the sequential revision process we have described is myopic. Another way of saying this is to describe the revision process we have defined as a markovian process: at each stage, the only information taken into account to revise is the state of the system at that stage. An alternative, more demanding, way of modelling things would be to go back, after each round of announcement, to the initial MKS and use all the sequence of announcements made up to that point in time to revise it. It is not clear whether the framework developed here is the most appropriate to treat this way of revising. Further, the "unbounded" memory assumption that this alternative approach would require might be too demanding in terms of the amount of information agents would have to keep at each stage of the revision process. Indeed, it is not necessary for totally correct MKS. Here again, an intuition that is correct in the absence of mistakes 
(i.e., the path through which one arrives at a given state of the epistemic system is not relevant) appears to be misleading in the more general case. Finally, non commutativity points out the fact that communication has another strategic aspect to it beyond its mere content: the order of the agenda (i.e., who gets to speak when) is important and agents are bound to take this into account if they have the choice as to when to speak.

Beyond these general remarks, we would like to argue that there is an important difference between the two examples of non-commutativity. In Example 33, noncommutativity is problematic: for instance, in the case where agents 1 and 2 announce first and then 3 announces, agent 1 should be allowed to reconsider the elimination of state $\omega_{2}$, since 2's and 3's announcements have essentially the same value to agent 1. The situation in Example 34 is different: the mistakes were not on the first level beliefs but on higher order beliefs. Hence, these beliefs do change after a first round of announcement. Thus, the non-commutativity of the rule simply reflects that higher order mistaken beliefs are corrected according to the announcements made at a given stage, before further revision is done.

\section{Appendix A: Irreducibility and Revision of MKS}

In this appendix, we tackle the issue of whether the revision process we defined depend on the representation of the MKS we consider. We first establish that if an MKS is correct so must be any representation of it.

Proposition 35. - Let $\left(\Omega, \omega_{0}, s,\left(t_{i}\right)_{i \in I}\right)$ and $\left(\Omega^{\prime}, \omega_{0}^{\prime}, s^{\prime},\left(t_{i}^{\prime}\right)_{i \in I}\right)$ be irreducible and equivalent MKS. If $\Omega$ is correct then, $\Omega^{\prime}$ is also correct.

In the last proposition of this appendix, we show that the sequential revision process does not depend on the choice of an irreducible representation at each stage. We first need to define a notion of compatibility of an agent's ordering between two equivalent irreducible MKS.

Definition 36. - Let $\left(\Omega, \omega_{0}, s,\left(t_{i}\right)_{i \in I}\right)$ and $\left(\Omega^{\prime}, \omega_{0}^{\prime}, s^{\prime},\left(t_{i}^{\prime}\right)_{i \in I}\right)$ be two irreducible and equivalent MKS. Two functions f from $\Omega$ to $2^{\Omega}$ and $f^{\prime}$ from $\Omega^{\prime}$ to $2^{\Omega^{\prime}}$ are compatible if there exists a function $\phi: \Omega \rightarrow \Omega^{\prime}$ such that that conditions (i) to (iv) of Definition 6 hold and such that $\forall \omega \in \Omega, f^{\prime}(\phi(\omega))=\phi(f(\omega))$.

Proposition 37. - Let $\left(\Omega, \omega_{0}, s,\left(t_{i}\right)_{i \in I}\right)$ and $\left(\Omega^{\prime}, \omega_{0}^{\prime}, s^{\prime},\left(t_{i}^{\prime}\right)_{i \in I}\right)$ be two irreducible equivalent MKS. Assume that agents' selection functions are compatible.

Then $\left(\Omega^{c}, \omega_{0}, s,\left(t_{i}^{c}\right)_{i \in I}\right)$ and $\left(\left(\Omega^{\prime}\right)^{c}, \omega_{0}^{\prime},\left(s^{\prime}\right),\left(\left(t_{i}^{\prime}\right)^{c}\right)_{i \in I}\right)$ are equivalent $M K S$.

\section{Appendix B: Proofs}

Proof (Proposition 2). - Assume $(v)$ and define the set $\Omega^{\prime} \subseteq \Omega$ by

$$
\begin{aligned}
& \Omega^{\prime}= \\
& \left\{\omega_{0}\right\} \cup\left\{\omega \in \Omega \mid \exists r \in \mathbb{N}, \exists\left\{i_{k}\right\}_{k=1}^{k=r}, i_{k} \in I, i_{r}=i \text { s.th. } \omega \in t_{i_{1}}\left(t_{i_{2}}\left(\ldots\left(t_{i_{r}}\left(\omega_{0}\right)\right)\right)\right\}\right.
\end{aligned}
$$


We show that $\left(\Omega^{\prime}, \omega_{0},\left.s\right|_{\Omega^{\prime}},\left(\left.t_{i}\right|_{\Omega^{\prime}}\right)_{i \in I}\right)$ satisfies conditions $(i)$ to $(i v)$ of Definition 1 . Conditions (i), (iii), and (iv) are obvious. Consider $i \in I, \omega \in \Omega^{\prime}$ and take $\omega^{\prime} \in$ $\left.t_{i}\right|_{\Omega^{\prime}}(\omega)=t_{i}(\omega)$. It is easy to see that by definition of $\Omega^{\prime}, \omega^{\prime} \in \Omega^{\prime}$ which proves that $\left.t_{i}\right|_{\Omega^{\prime}}$ is a mapping from $\Omega^{\prime}$ to $2^{\Omega^{\prime}}$. Therefore, condition $(v)$ implies that $\Omega^{\prime}=\Omega$ and thus condition $\left(v^{\prime}\right)$ holds.

Assume now $\left(v^{\prime}\right)$ and suppose there exists $\Omega^{\prime} \varsubsetneqq \Omega$ such that $\left(\Omega^{\prime}, \omega_{0},\left.s\right|_{\Omega^{\prime}},\left(\left.t_{i}\right|_{\Omega^{\prime}}\right)_{i \in I}\right)$ satisfy conditions $(i)$ to $(i v)$ of Definition 1 . Hence, $\exists \omega \in \Omega \backslash \Omega^{\prime}$. However by $\left(v^{\prime}\right)$, $\exists r \in \mathbb{N}$ and $\left\{i_{k}\right\}_{k=1}^{k=r}, i_{k} \in I, i_{r}=i$ s.th. $\omega \in t_{i_{1}}\left(t_{i_{2}}\left(\ldots\left(t_{i_{r}}\left(\omega_{0}\right)\right)\right)\right.$. Since $\omega_{0} \in \Omega^{\prime}$, then $\left.t_{i_{r}}\right|_{\Omega^{\prime}}\left(\omega_{0}\right)=t_{i_{r}}\left(\omega_{0}\right) \subseteq \Omega^{\prime}$ since condition (ii) applies. By induction, we can show that for all $k=1, . . r$,

$$
\left(\left.t_{i_{k}}\right|_{\Omega^{\prime}}\left(\ldots\left(\left.t_{i_{r}}\right|_{\Omega^{\prime}}\left(\omega_{0}\right)\right)\right)=\left(t_{i_{k}}\left(\ldots\left(t_{i_{r}}\left(\omega_{0}\right)\right)\right) \subseteq \Omega^{\prime}\right.\right.
$$

and thus $\omega \in \Omega^{\prime}$ yielding a contradiction.

\section{ProOF (PROPOSITION 10). - -}

Let $R(\Omega)$ be the set of representations of $\Omega$, i.e., the set of $\operatorname{MKS}\left(\Omega^{\prime}, \omega_{0}^{\prime}, s^{\prime},\left(t_{i}^{\prime}\right)_{i \in I}\right)$ such that there exists a mapping $\sigma$ from $\Omega$ to $\Omega^{\prime}$ that satisfies the properties of Definition 6.

Let $\bar{\sigma}$ be a mapping from $\Omega$ to $\Omega$ that satisfies $\bar{\sigma}\left(\omega_{1}\right)=\bar{\sigma}\left(\omega_{2}\right)$ if and only if there exists an MKS $\left(\Omega^{\prime}, \omega_{0}^{\prime}, s^{\prime},\left(t_{i}^{\prime}\right)_{i \in I}\right)$ and a mapping $\sigma$ from $\Omega$ to $\Omega^{\prime}$ that satisfies the properties of Definition 6 such that $\sigma\left(\omega_{1}\right)=\sigma\left(\omega_{2}\right)$. Let $\bar{\Omega}=\bar{\sigma}(\Omega)$ and $\bar{\omega}_{0}=\bar{\sigma}\left(\omega_{0}^{\prime}\right)$.

Define $\bar{s}: \bar{\Omega} \rightarrow S$ by $\bar{s}(\bar{\omega})=s\left(\omega_{1}\right)$ where $\omega_{1} \in \Omega$ is such that $\bar{\sigma}\left(\omega_{1}\right)=\bar{\omega}$. This is well defined since if $\bar{\sigma}\left(\omega_{1}\right)=\bar{\sigma}\left(\omega_{2}\right)$ we know that there exists $\sigma$ such that $\sigma\left(\omega_{1}\right)=\sigma\left(\omega_{2}\right)$ which implies that $s\left(\omega_{1}\right)=s\left(\omega_{2}\right)$ since $\Omega^{\prime}$ is a representation of $\Omega$ via $\sigma$.

Next, we show that if $\bar{\sigma}\left(\omega_{1}\right)=\bar{\sigma}\left(\omega_{2}\right)$ then $\bar{\sigma}\left(t_{i}\left(\omega_{1}\right)\right)=\bar{\sigma}\left(t_{i}\left(\omega_{2}\right)\right)$. Since $\bar{\sigma}\left(\omega_{1}\right)=\bar{\sigma}\left(\omega_{2}\right)$, it must be the case that there exists $\sigma$ such that $\sigma\left(\omega_{1}\right)=\sigma\left(\omega_{2}\right)$. Then, $\sigma\left(t_{i}\left(\omega_{1}\right)\right)=\sigma\left(t_{i}\left(\omega_{2}\right)\right)$. Now, let $\bar{\omega} \in \bar{\sigma}\left(t_{i}\left(\omega_{1}\right)\right)$. There exists $\omega_{3} \in t_{i}^{\prime}\left(\omega_{1}\right)$ such that $\bar{\sigma}\left(\omega_{3}\right)=\bar{\omega}$. Since $\sigma\left(\omega_{3}\right) \in \sigma\left(t_{i}\left(\omega_{1}\right)\right)=\sigma\left(t_{i}\left(\omega_{2}\right)\right)$, there exists $\omega_{4} \in$ $t_{i}\left(\omega_{2}\right)$ such that $\sigma\left(\omega_{3}\right)=\sigma\left(\omega_{4}\right)$. Hence, $\bar{\sigma}\left(\omega_{3}\right)=\bar{\sigma}\left(\omega_{4}\right) \in \bar{\sigma}\left(t_{i}\left(\omega_{2}\right)\right)$ and therefore $\bar{\omega} \in \bar{\sigma}\left(t_{i}\left(\omega_{2}\right)\right)$ proving that $\bar{\sigma}\left(t_{i}\left(\omega_{1}\right)\right) \subseteq \bar{\sigma}\left(t_{i}\left(\omega_{2}\right)\right)$. Similarly, the reverse inclusion holds and hence $\bar{\sigma}\left(t_{i}\left(\omega_{1}\right)\right)=\bar{\sigma}\left(t_{i}\left(\omega_{2}\right)\right)$.

Finally, define $\bar{t}_{i}: \bar{\Omega} \rightarrow 2^{\bar{\Omega}}$ by $\bar{t}_{i}(\bar{\omega})=\bar{t}_{i}(\bar{\sigma}(\omega))=\bar{\sigma}\left(t_{i}(\omega)\right)$ where $\omega \in \Omega$ is such that $\bar{\sigma}(\omega)=\bar{\omega}$. This is well defined since we showed that if $\bar{\omega}$ has two antecedents $\omega_{1}$ and $\omega_{2}, \bar{\sigma}\left(t_{i}\left(\omega_{1}\right)\right)=\bar{\sigma}\left(t_{i}\left(\omega_{2}\right)\right)$.

We first show that $\left(\bar{\Omega}, \bar{\omega}_{0}, \bar{s},\left(\bar{t}_{i}\right)_{i \in I}\right)$ so defined is an MKS. The two conditions to check are condition $($ iii) and $(v)$ of Definition 1. Check first condition $($ iii $)$ and let $\bar{\omega}_{2} \in \bar{t}_{i}\left(\bar{\omega}_{1}\right)$. There exist $\omega_{1}$ and $\omega_{2}$ such that $\bar{\sigma}\left(\omega_{1}\right)=\bar{\omega}_{1}$ and $\bar{\sigma}\left(\omega_{2}\right)=\bar{\omega}_{2}$ and $\omega_{2} \in t_{i}\left(\omega_{1}\right)$. Hence $t_{i}\left(\omega_{1}\right)=t_{i}\left(\omega_{2}\right)$ and therefore $\bar{\sigma}\left(t_{i}\left(\omega_{1}\right)\right)=\bar{\sigma}\left(t_{i}\left(\omega_{2}\right)\right)$, i.e., $\bar{t}_{i}\left(\bar{\omega}_{1}\right)=\bar{t}_{i}\left(\bar{\omega}_{2}\right)$. 
We next check that condition $\left(v^{\prime}\right)$ holds (by Proposition 2, this is equivalent to check condition $(v)$ of Definition 1 directly). Let $\bar{\omega} \in \bar{\Omega}$. By construction, there exists $\omega \in \Omega$ such that $\bar{\sigma}(\omega)=\bar{\omega}$. Thus, there exists $r$ finite and a sequence $\left\{i_{k}\right\}_{k=1}^{k=r}$ with $i_{k} \in I$ for all $i$ such that $\omega \in t_{i_{1}}\left(t_{i_{2}}\left(\ldots\left(t_{i_{r}}\left(\omega_{0}\right)\right)\right)\right)$. Hence,

$$
\bar{\sigma}(\omega) \in \bar{\sigma}\left[t_{i_{1}}\left(t_{i_{2}}\left(\ldots\left(t_{i_{r}}\left(\omega_{0}\right)\right)\right)\right)\right]
$$

Recall that $\bar{\sigma}\left(t_{i}(\omega)\right)=\bar{t}_{i}(\bar{\sigma}(\omega))$. Hence,

$$
\bar{\sigma}\left[t_{i_{1}}\left(t_{i_{2}}\left(\ldots\left(t_{i_{r}}\left(\omega_{0}\right)\right)\right)\right)\right]=\bar{t}_{i_{1}}\left(\bar{\sigma}\left[t_{i_{2}}\left(\ldots\left(t_{i_{r}}\left(\omega_{0}\right)\right)\right)\right]\right)
$$

and, eventually,

$$
\bar{\sigma}\left[t_{i_{1}}\left(t_{i_{2}}\left(\ldots\left(t_{i_{r}}\left(\omega_{0}\right)\right)\right)\right)\right]=\bar{t}_{i_{1}}\left(\bar{t}_{i_{2}}\left(\ldots\left(\bar{t}_{i_{r}}\left(\bar{\sigma} \omega_{0}\right)\right)\right)\right)=\bar{t}_{i_{1}}\left(\bar{t}_{i_{2}}\left(\ldots\left(\bar{t}_{i_{r}}\left(\bar{\omega}_{0}\right)\right)\right)\right)
$$

proving condition $\left(v^{\prime}\right)$ of Proposition 2. Observe that $\left(\bar{\Omega}, \bar{\omega}_{0}, \bar{s},\left(\bar{t}_{i}\right)_{i \in I}\right)$ is a representation of $\left(\Omega, \omega_{0}, s,\left(t_{i}\right)_{i \in I}\right)$, since $\bar{\sigma}$ satisfies the conditions of Definition 6.

We next want to show that $\left(\bar{\Omega}, \bar{\omega}_{0}, \bar{s},\left(\bar{t}_{i}\right)_{i \in I}\right)$ is irreducible. Assume this is not the case and that there exists a representation $\left(\widetilde{\Omega}, \widetilde{\omega}_{0}, \widetilde{s},\left(\widetilde{t}_{i}\right)_{i \in I}\right)$ of $\left(\bar{\Omega}, \bar{\omega}_{0}, \bar{s},\left(\bar{t}_{i}\right)_{i \in I}\right)$ and a mapping $\widetilde{\sigma}: \widetilde{\Omega} \rightarrow \bar{\Omega}$ such that $\widetilde{\sigma}\left(\bar{\omega}_{1}\right)=\widetilde{\sigma}\left(\bar{\omega}_{2}\right)$ for some $\bar{\omega}_{1}, \bar{\omega}_{2} \in \bar{\Omega}, \bar{\omega}_{1} \neq \bar{\omega}_{2}$. Let $\omega_{1}$ and $\omega_{2}$ in $\Omega$ be such that $\bar{\omega}_{1}=\bar{\sigma}\left(\omega_{1}\right)$ and $\bar{\omega}_{2}=\bar{\sigma}\left(\omega_{2}\right)$. It is easy to show that $\left(\widetilde{\Omega}, \widetilde{\omega}_{0}, \widetilde{s},\left(\widetilde{t}_{i}\right)_{i \in I}\right)$ is also a representation of $\left(\Omega, \omega_{0}, s,\left(t_{i}\right)_{i \in I}\right)$ via the mapping $\widetilde{\sigma} \circ \bar{\sigma}$. Hence, $\widetilde{\Omega} \in R(\Omega)$ and $\bar{\sigma}\left(\omega_{1}\right)=\bar{\sigma}\left(\omega_{2}\right)$, i.e., $\bar{\omega}_{1}=\bar{\omega}_{2}$, a contradiction.

ProOf (Propositions 14 AND 16). - First, we prove the following lemma:

Lemma 38. - Let $\left(\Omega, \omega_{0}, s,\left(t_{i}\right)_{i \in I}\right)$ be an MKS. For all $i \in I, \forall \omega \in \Omega$,

$\omega \in B H_{i}\left(\omega_{0}, t\right) \Leftrightarrow \exists r \in \mathbb{N}, \exists\left\{i_{k}\right\}_{k=1}^{k=r}, i_{k} \in I, i_{r}=i$ s.th. $\omega \in t_{i_{1}}\left(t_{i_{2}}\left(\ldots\left(t_{i_{r}}\left(\omega_{0}\right)\right)\right)\right.$

Proof (Lemma 38). - For $i \in I$ consider

$N H_{i}\left(\omega_{0}, t\right)=$

$\left\{\omega \in B H_{i}\left(\omega_{0}, t\right) \mid \forall r \in \mathbb{N}\right.$ and $\left\{i_{k}\right\}_{k=1}^{k=r}, i_{k} \in I, i_{r}=i$ s.th. $\omega \notin t_{i_{1}}\left(t_{i_{2}}\left(\ldots\left(t_{i_{r}}\left(\omega_{0}\right)\right)\right)\right\}$

and suppose $N H_{i}\left(\omega_{0}, t\right) \neq \emptyset$. Consider

$$
Y=B H_{i}\left(\omega_{0}, t\right) \backslash N H_{i}\left(\omega_{0}, t\right)
$$

Note that $Y$ is strictly included in $B H_{i}\left(\omega_{0}, t\right)$ since $N H_{i}\left(\omega_{0}, t\right) \neq \emptyset$. Remark that trivially $t_{i}\left(\omega_{0}\right) \subseteq Y$ which shows that $Y \neq \emptyset$ and condition $(i)$ of Definition 13 is satisfied. 
Consider $\omega^{\prime} \in Y$ and $j \in I$. Since $\omega^{\prime} \in B H_{i}\left(\omega_{0}, t\right), t_{j}\left(\omega^{\prime}\right) \subseteq B H_{i}\left(\omega_{0}, t\right)$. Suppose that $t_{j}\left(\omega^{\prime}\right) \nsubseteq Y$ and thus there exists $\omega \in N H_{i}\left(\omega_{0}, t\right) \cap t_{j}\left(\omega^{\prime}\right)$. Since $\omega^{\prime} \in Y$, there exists a sequence $\left\{i_{k}\right\}_{k=1}^{k=r}, i_{k} \in I, i_{r}=i$ such that $\omega^{\prime} \in t_{i_{1}}\left(t_{i_{2}}\left(\ldots\left(t_{i_{r}}\left(\omega_{0}\right)\right)\right)\right.$. Then define the sequence $\left\{i_{k}^{\prime}\right\}_{k=1}^{k=r+1}$ by $i_{1}^{\prime}=j, i_{k}^{\prime}=i_{k-1}$ for all $k=2, . ., r+1$. Note that $i_{r+1}^{\prime}=i$. Then we have that $\omega \in t_{i_{1}^{\prime}}\left(t_{i_{2}^{\prime}}\left(\ldots\left(t_{i_{r+1}^{\prime}}\left(\omega_{0}\right)\right)\right)\right.$ which is a contradiction with $\omega \in N H_{i}\left(\omega_{0}, t\right)$. Thus, condition (ii) of Definition 13 is also satisfied. That proves that $B H_{i}\left(\omega_{0}, t\right)$ is not the irreducible subset which satisfies these conditions.

Thus $N H_{i}\left(\omega_{0}, t\right)=\emptyset$ and

$$
\begin{gathered}
B H_{i}\left(\omega_{0}, t\right) \subseteq \\
\left\{\omega \in \Omega \mid \exists r \in \mathbb{N} \text { and }\left\{i_{k}\right\}_{k=1}^{k=r}, i_{k} \in I, i_{r}=i \text { s.th. } \omega \in t_{i_{1}}\left(t_{i_{2}}\left(\ldots\left(t_{i_{r}}\left(\omega_{0}\right)\right)\right)\right\}\right.
\end{gathered}
$$

Conversely, consider $\omega \in \Omega$ such that there exists ${ }^{10} r \in \mathbb{N}$ and $\left\{i_{k}\right\}_{k=1}^{k=r}, i_{k} \in I$, $i_{r}=i$ such that $\omega \in t_{i_{1}}\left(t_{i_{2}}\left(\ldots\left(t_{i_{r}}\left(\omega_{0}\right)\right)\right)\right.$ and let us suppose that $\omega \notin B H_{i}\left(\omega_{0}, t\right)$. Then there exists $\left\{\omega_{k}\right\}_{k=1}^{k=r}$ such that $\omega_{r}=\omega_{0}, \forall k=1, . ., r-1 \omega_{k} \in t_{i_{k+1}}\left(\omega_{k+1}\right)$ and $\omega \in t_{i_{1}}\left(\omega_{1}\right)$. Since $\omega \notin B H_{i}\left(\omega_{0}, t\right)$, condition (ii) of Definition 13 implies that $\omega_{1} \notin$ $B H_{i}\left(\omega_{0}, t\right)$. Recursively, we have that $\forall k=1, . ., r-1, \omega_{k} \notin B H_{i}\left(\omega_{0}, t\right)$. Hence, since $\omega_{r-1} \notin B H_{i}\left(\omega_{0}, t\right), t_{i}\left(\omega_{0}\right) \nsubseteq B H_{i}\left(\omega_{0}, t\right)$, contradicting $(i)$ of Definition 13 .

Propositions 14 and 16 are direct consequences of the lemma.

Proof (Proposition 23). - It is straightforward to check conditions $(i)$ to $(v)$ of Definition 1 for both systems.

PROOF (PROPOSITION 25). -

Before proceeding to the proof of the proposition itself, we need a lemma in which CSBS is characterized by the fact that any given agent must have the same beliefs in all the states of the world.

Lemma 39. - Let $\left(\Omega, \omega_{0}, s,\left(t_{i}\right)_{i \in I}\right)$ be an irreducible MKS. Then, the following assertions are equivalent

(i) $\left(\Omega, \omega_{0}, s,\left(t_{i}\right)_{i \in I}\right)$ is $C S B S$

(ii) $S B\left(\omega_{0}, t\right)=\Omega$

(iii) $\forall \omega \in \Omega, \forall i \in I, \quad t_{i}(\omega)=t_{i}\left(\omega_{0}\right)$

Proof (Lemma 39). - We first prove $(i) \Leftrightarrow(i i)$. Since $S B\left(\omega_{0}, t\right)$ is common beliefs, we know by Corollary 17 that $\cup_{i \in I} B H_{i}\left(\omega_{0}, t\right) \subseteq S B\left(\omega_{0}, t\right) \subseteq \Omega=\left\{\omega_{0}\right\} \cup_{i \in I}$ $B H_{i}\left(\omega_{0}, t\right)$. Note that by definition, $\omega_{0} \in S B\left(\omega_{0}, t\right)$ and thus $S B\left(\omega_{0}, t\right)=\Omega$. Conversely, if $S B\left(\omega_{0}, t\right)=\Omega$, then $\left(\Omega, \omega_{0}, s,\left(t_{i}\right)_{i \in I}\right)$ is a CSBS.

10. By Proposition 2, such an $r$ exists. 
We next prove $(i) \Leftrightarrow(i i i)$. From what we just proved, one way is obvious: since the condition $t_{i}(\omega)=t_{i}\left(\omega_{0}\right) \forall \omega \in \Omega, \forall i \in I$ implies that $S B\left(\omega_{0}, t\right)=\Omega$, and hence the MKS is CSBS.

Conversely, assume that the MKS is CSBS. Then $S B\left(\omega_{0}, t\right)=\Omega$. Consider $\Omega^{\prime}=$ $\left\{\omega_{s}^{\prime}\right\}_{s \in s(\Omega)}$ and the MKS, $\left(\Omega^{\prime}, \omega_{s\left(\omega_{0}\right)}^{\prime}, s^{\prime},\left(t_{i}^{\prime}\right)_{i \in I}\right)$ defined by $\forall \omega_{s}^{\prime} \in \Omega^{\prime}, s^{\prime}\left(\omega_{s}^{\prime}\right)=s$ and $\forall i \in I, t_{i}^{\prime}\left(\omega_{s}^{\prime}\right)=\left\{\omega_{s^{\prime}}^{\prime} \in \Omega \mid s^{\prime} \in s\left(t_{i}\left(\omega_{0}\right)\right)\right\}$. Define the mapping $\sigma: \Omega \rightarrow \Omega^{\prime}$ by $\forall \omega \in \Omega, \sigma(\omega)=\omega_{s(\omega)}^{\prime}$. By construction, we have that $\sigma(\Omega)=\Omega^{\prime}, \sigma\left(\omega_{0}\right)=\omega_{s\left(\omega_{0}\right)}^{\prime}$, and $s^{\prime} \circ \sigma=s$. Consider now $i \in I$ and $\omega \in \Omega$. Then

$$
t_{i}^{\prime} \circ \sigma(\omega)=t_{i}^{\prime}\left(\omega_{s(\omega)}^{\prime}\right)=\left\{\omega_{s^{\prime}}^{\prime} \in \Omega \mid s^{\prime} \in s\left(t_{i}\left(\omega_{0}\right)\right)\right\}
$$

while

$$
\begin{aligned}
\sigma \circ t_{i}(\omega) & =\left\{\omega_{s^{\prime}}^{\prime} \in \Omega \mid \exists \omega^{\prime \prime} \in t_{i}(\omega) \text { such that } \omega_{s^{\prime}}^{\prime}=\sigma\left(\omega^{\prime \prime}\right)\right\} \\
& =\left\{\omega_{s^{\prime}}^{\prime} \in \Omega \mid \exists \omega^{\prime \prime} \in t_{i}(\omega) \text { such that } s^{\prime}=s\left(\omega^{\prime \prime}\right)\right\} \\
& =\left\{\omega_{s^{\prime}}^{\prime} \in \Omega \mid s^{\prime} \in s\left(t_{i}(\omega)\right)\right\}
\end{aligned}
$$

But since $S B\left(\omega_{0}, t\right)=\left\{\omega \in \Omega \mid s\left(t_{i}(\omega)\right)=s\left(t_{i}\left(\omega_{0}\right)\right) \forall i \in I\right\}=\Omega$, we have

$$
\sigma \circ t_{i}(\omega)=\left\{\omega_{s^{\prime}}^{\prime} \in \Omega \mid s^{\prime} \in s\left(t_{i}\left(\omega_{0}\right)\right)\right\}=t_{i}^{\prime} \circ \sigma(\omega)
$$

Thus $t_{i}^{\prime} \circ \sigma=\sigma \circ t_{i}$ which shows that the MKS, $\left(\Omega^{\prime}, \omega_{s\left(\omega_{0}\right)}, s^{\prime},\left(t_{i}^{\prime}\right)_{i \in I}\right)$ is a representation of the MKS, $\left(\Omega, \omega_{0}, s,\left(t_{i}\right)_{i \in I}\right)$. Since $\left(\Omega, \omega_{0}, s,\left(t_{i}\right)_{i \in I}\right)$ is irreducible, $\sigma$ is a one-to-one mapping. Remark now that by construction $\forall \omega_{s}^{\prime} \in \Omega^{\prime}, \forall i \in I, t_{i}\left(\omega_{s}^{\prime}\right)=$ $t_{i}\left(\omega_{s\left(\omega_{0}\right)}^{\prime}\right)$ and since $\sigma^{-1}$ is a one-to-one mapping, $t_{i}\left(\sigma^{-1}\left(\omega_{s}^{\prime}\right)\right)=t_{i}\left(\sigma^{-1}\left(\omega_{s\left(\omega_{0}\right)}^{\prime}\right)\right)$, establishing that $\forall \omega \in \Omega, \forall i \in I, t_{i}(\omega)=t_{i}\left(\omega_{0}\right)$.

The proof of Proposition 25 is now trivial: If $I^{c}=I$, then by the construction of $t_{i}^{c}$ given in Definition 20, $\forall i, \forall \omega \in \Omega^{c}, t_{i}^{c}(\omega)=f_{i}\left(\omega_{0}\right)=t_{i}^{c}\left(\omega_{0}\right)$ and thus according to Proposition $39,\left(\Omega^{c}, \omega_{0}, s,\left(t_{i}^{c}\right)_{i \in I}\right)$ is a CSBS.

Proof (Proposition 26). - Since $\left(\Omega, \omega_{0}, s,\left(t_{i}\right)_{i \in I}\right)$ is a CSBS, Lemma 39 yields that $\forall \omega \in \Omega, \forall i \in I, \quad t_{i}(\omega)=t_{i}\left(\omega_{0}\right)$. Hence, $\Omega=\left\{\omega \in \Omega \mid t_{j}(\omega)=\right.$ $\left.t_{j}\left(\omega_{0}\right), \forall j \in I^{c}\right\}$ and hence, given that the selection functions have the consistency property, $\forall \omega \in \Omega, \forall i, t_{i}^{c}(\omega)=t_{i}\left(\omega_{0}\right)$ and thus

$$
\Omega^{c}=\left\{\omega_{0}\right\} \cup\left(\cup_{i \in I} B H_{i}\left(\omega_{0}, t^{c}\right)\right)=\Omega
$$

which establishes that $\left(\Omega^{c}, \omega_{0}, s,\left(t_{i}^{c}\right)_{i \in I}\right)=\left(\Omega, \omega_{0}, s,\left(t_{i}\right)_{i \in I}\right)$.

Proof (Proposition 28). $-\Omega^{c}$ is consensual if and only if $f_{i}\left(\omega_{0}\right)=f_{j}\left(\omega_{0}\right)$ for all $i, j \in I$. Given that $\left\{\omega \mid t_{i}(\omega)=t_{i}\left(\omega_{0}\right) \forall i \in I\right\} \cap t_{i}\left(\omega_{0}\right) \neq \emptyset, \Omega^{c}$ is consensual if and only if 
$t_{i}\left(\omega_{0}\right) \cap\left\{\omega \mid t_{i}(\omega)=t_{i}\left(\omega_{0}\right) \forall i \in I\right\}=t_{j}\left(\omega_{0}\right) \cap\left\{\omega \mid t_{i}(\omega)=t_{i}\left(\omega_{0}\right) \forall i \in I\right\} \quad \forall i, j \in I$.

$[\Rightarrow]$ Assume $\Omega^{c}$ consensual.

It is easy to check that if $\cap_{i \in I} t_{i}\left(\omega_{0}\right) \neq \emptyset$, then $\cap_{i \in I} t_{i}\left(\omega_{0}\right)=\left\{\omega \mid t_{i}(\omega)=\right.$ $\left.t_{i}\left(\omega_{0}\right), \forall i \in I\right\}$.

Since for all $i, j \in I$,

$$
t_{i}\left(\omega_{0}\right) \cap\left\{\omega \mid t_{i}(\omega)=t_{i}\left(\omega_{0}\right) \forall i \in I\right\}=t_{j}\left(\omega_{0}\right) \cap\left\{\omega \mid t_{i}(\omega)=t_{i}\left(\omega_{0}\right) \forall i \in I\right\} \neq \emptyset
$$

we get that $\cap_{i \in I} t_{i}\left(\omega_{0}\right) \neq \emptyset$. Therefore, $t_{i}\left(\omega_{0}\right) \cap\left\{\omega \mid t_{i}(\omega)=t_{i}\left(\omega_{0}\right) \forall i \in I\right\}=$ $t_{i}\left(\omega_{0}\right) \cap_{i \in I} t_{i}\left(\omega_{0}\right)=\cap_{i \in I} t_{i}\left(\omega_{0}\right)$

$[\Leftarrow]$ Assume $t_{i}\left(\omega_{0}\right) \cap\left\{\omega \mid t_{j}(\omega)=t_{j}\left(\omega_{0}\right) \forall j \in I\right\}=\cap_{i \in I} t_{i}\left(\omega_{0}\right)$. Then, trivially, $t_{i}\left(\omega_{0}\right) \cap\left\{\omega \mid t_{i}(\omega)=t_{i}\left(\omega_{0}\right) \forall i \in I\right\}=t_{j}\left(\omega_{0}\right) \cap\left\{\omega \mid t_{i}(\omega)=t_{i}\left(\omega_{0}\right) \forall i \in I\right\} \quad \forall i, j \in I$ and $\Omega^{c}$ is consensual.

Proof (Proposition 30). - This is readily deduced from three observations:

- After a communication, if $i \in I^{c}$, then by definition, for all $\omega \in \Omega^{c}, t_{i}^{c}(\omega)=$ $t_{i}^{c}\left(\omega_{0}\right)$.

- If we start from a situation where the MKS is such that for $i, \forall \omega \in \Omega, t_{i}(\omega)=$ $t_{i}\left(\omega_{0}\right)$, then after a communication, it is also the case that $\forall \omega \in \Omega^{c}, t_{i}^{c}(\omega)=t_{i}^{c}\left(\omega_{0}\right)$.

- Reducing MKS at each stage to irreducible MKS if necessary, does not affect the two previous properties.

Thus if the sequential communication is exhaustive, we have that $\forall \omega \in \Omega_{T}^{c}$, $t_{i, T}^{c}(\omega)=t_{i, T}^{c}\left(\omega_{0}\right)$ which characterizes CSBS.

ProOF (Proposition 31). - We prove the proposition by induction. It is trivial that the proposition is true for $\tau=1$ since the two communications are identical.

Let now $\tau \geq 2$ and assume that for all $\iota=1, \ldots, \tau-1,\left(\Omega_{\iota}^{c}, \omega_{0}, s,\left(t_{i, \iota}^{c}\right)_{i \in I}\right)_{\iota=1, \ldots, \tau-1}$ is a sequence of revised MKS for communication $\left(I_{\iota}^{c}\right)_{\iota=1, \ldots, \tau-1}$.

Consider $\left(f_{i, \tau}\right)$, the selection functions at stage $\tau$ for communication $I^{c}$. Observe that these are also selection functions at stage $\tau$ for communication $\bar{I}^{c}$. Indeed, conditions $(i)$ and $(i i)$ of definition 19 are satisfied. To prove condition (iii), consider $j \in I_{\tau}^{c} \backslash \bar{I}_{\tau}^{c}$. Then, there exists $\iota \leq \tau-1$ such that $j \in I_{\iota}^{c}$. According to the revision rule, we have $t_{j, \iota}^{c}(\omega)=t_{j, \iota}^{c}\left(\omega_{0}\right)$ for all $\omega \in \Omega_{\iota}^{c}$. Then, it is easy to see that for all $\kappa=\iota, \ldots, \tau-1$, we also have $t_{j, \kappa}^{c}(\omega)=t_{j, \kappa}^{c}\left(\omega_{0}\right)$ for all $\omega \in \Omega_{\kappa}^{c}$.

Hence, for all $j \in I_{\tau}^{c} \backslash \bar{I}_{\tau}^{c}, t_{j, \tau-1}^{c}(\omega)=t_{j, \tau-1}^{c}\left(\omega_{0}\right)$ for all $\omega \in \Omega_{\tau-1}^{c}$ and hence, $\left\{\omega \in \Omega_{\tau-1}^{c} \mid t_{j, \tau-1}^{c}(\omega)=t_{j, \tau-1}^{c}\left(\omega_{0}\right) \forall j \in I_{\tau}^{c}\right\}=\left\{\omega \in \Omega_{\tau-1}^{c} \mid t_{j, \tau-1}^{c}(\omega)=t_{j, \tau-1}^{c}\left(\omega_{0}\right) \forall j \in \bar{I}_{\tau}^{c}\right\}$ which proves that the selection functions are consistent for communication $\bar{I}^{c}$. 
Since for all $j \in I_{\tau}^{c} \backslash \bar{I}_{\tau}^{c}, t_{j, \tau-1}^{c}(\omega)=t_{j, \tau-1}^{c}\left(\omega_{0}\right)$ for all $\omega \in \Omega_{\tau-1}^{c}$, it is easy to check that applying the revision rule according to the selection functions $\left(f_{i, \tau}\right)$ for communication $I_{\tau}^{c}$ or for communication $\bar{I}_{\tau}^{c}$ leads to the same result.

Proof (PROPOSITION 32). - This is readily deduced from the following observations:

- When the MKS is totally correct the revision rule can be applied even if the MKS is not irreducible. It yields the same MKS as if it were applied on the irreducible MKS to begin with.

- At each stage, the revised MKS is totally correct.

- Therefore, revision can be done without worrying about irreducibility of the MKS.

- Thus, the MKS eventually reached corresponds to taking the intersection of all the agents' announcements, an operation that does not depend on the order of these announcements.

Proof (Proposition 35). - Observe that for all $i \in I, t_{i}^{\prime}(\sigma(\omega))=\sigma\left(t_{i}(\omega)\right)$ by construction and $\omega \in t_{i}(\omega)$ by assumption. Hence, $\sigma(\omega) \in \sigma\left(t_{i}(\omega)\right)$ and therefore $\sigma(\omega) \in t_{i}^{\prime}(\sigma(\omega))$ for all $i \in I$.

ProOF (PRoposition 37). -

First, we prove the following lemma:

LEMma 40. - Let $\left(\Omega, \omega_{0}, s,\left(t_{i}\right)_{i \in I}\right)$ and $\left(\Omega^{\prime}, \omega_{0}^{\prime}, s^{\prime},\left(t_{i}^{\prime}\right)_{i \in I}\right)$ be irreducible and equivalent MKS. Then there exists a one-to-one and onto mapping $\phi$ from $\Omega$ to $\Omega^{\prime}$ such that conditions (i) to (iv) of Definition 6 hold.

Proof (Lemma 40). - By Proposition 10, $\Omega$ and $\Omega^{\prime}$ have a common irreducible representation $\Omega$ ". Let $\sigma: \Omega \rightarrow \Omega$ " and $\sigma^{\prime}: \Omega^{\prime} \rightarrow \Omega$ " be the associated mappings. By definition, $\sigma$ and $\sigma^{\prime}$ are onto. Assume $\sigma$ is not one-to-one, i.e., there exist $\omega_{1}, \omega_{2} \in$ $\Omega, \omega_{1} \neq \omega_{2}$, such that $\sigma\left(\omega_{1}\right)=\sigma\left(\omega_{2}\right)$. This implies that $\Omega$ is not irreducible, a contradiction. Hence $\sigma$ is one-to-one. A similar argument holds for $\sigma^{\prime}$. Therefore, $\left(\sigma^{\prime}\right)^{-1} \circ \sigma$ is a well defined mapping from $\Omega$ to $\Omega^{\prime}$ that is one-to-one and onto. Take $\phi=\left(\sigma^{\prime}\right)^{-1} \circ \sigma$. Conditions (i) to (iv) hold by construction.

Let now $\phi$ be defined as in Lemma 40. Since the selection functions are compatible, it is easy to check that $t_{i}^{\prime^{c}} \circ \phi(\omega)=\phi \circ t_{i}^{c}(\omega)$ for all $\omega \in \Omega$. Hence, $\left(\left(\Omega^{\prime}\right)^{c}, \omega_{0}^{\prime}, s^{\prime},\left(\left(t_{i}^{\prime}\right)^{c}\right)_{i \in I}\right)$ is a representation of $\left(\Omega^{c}, \omega_{0}, s,\left(t_{i}^{c}\right)_{i \in I}\right)$. 


\section{References}

[ALC 85] Alchourron C., GÄrdenfors P., Makinson D., "On the Logic of Theory Change: Partial Meet Contraction and Revision Functions", The Journal of Symbolic Logic, vol. 50, 1985, p. 510-530.

[BOA 03] BOARD O., "Dynamic Interactive Epistemology”, Games and Economic Behavior, 2003, forthcoming.

[BON 96] Bonanno G., "On the Logic of Common Belief”, Mathematical Logic Quarterly, vol. 42, 1996, p. 305-311.

[CHE 80] Chellas B., Modal Logic: an introduction, Cambridge University Press, 1980.

[GER 97] Gerbrandy J., GROENEVELD W., "Reasoning about Information Change", Journal of Logic, Language, and Information, vol. 6, 1997, p. 147-169. 